\title{
Retraction Note: Aluminum in Neurological and Neurodegenerative Disease
}

\author{
Donald R. C. McLachlan ${ }^{1,2} \cdot$ Catherine Bergeron ${ }^{1,2} \cdot$ Peter N. Alexandrov ${ }^{3} \cdot$ William J. Walsh $^{4} \cdot$ Aileen I. Pogue $^{5}$. \\ Maire E. Percy ${ }^{1,6,7}$. Theodore P. A. Kruck ${ }^{1} \cdot$ Zhide Fang $^{8,9,10}$ • Nathan M. Sharfman ${ }^{11}$ • Vivian Jaber ${ }^{11}$ - Yuhai Zhao ${ }^{11,12}$. \\ Wenhong $\mathrm{Li}^{11,13} \cdot$ Walter J. Lukiw ${ }^{3,5,11,14,15}$
}

Published online: 23 January 2020

(C) Springer Science+Business Media, LLC, part of Springer Nature 2020

\section{Retraction Note: Molecular Neurobiology (2019) 56:1531-1538 https://doi.org/10.1007/s12035-018-1441-x}

The Editor-in Chief of Molecular Neurobiology has retracted this article [1] at the request of the corresponding author. This is because it significantly overlaps with their previous publication [2]. Both articles report the same results and as such this article is redundant.

Walter J. Lukiw, Maire E. Percy, and Zhide Fang agree to this retraction. William J. Walsh and Yuhai Zhao do not agree to this retraction. Aileen I. Pogue, Nathan M. Sharfman, Vivian Jaber, and Wenhong Li have not responded to any correspondence from the editor/publisher about this retraction. Donald R. C. McLachlan, Catherine Bergeron, Peter N. Alexandrov, and Theodore P. A. Kruck are deceased.

[1] McLachlan, D.R.C., Bergeron, C., Alexandrov, P.N. et al. Mol Neurobiol (2019) 56: 1531. https://doi.org/10. 1007/s12035-018-1441-x

[2] McLachlan, D.R.C., Alexandrov, P.N., Walsh, W.J. et al. J Alzheimers Dis Parkinsonism (2018) 8(6): 457. https://doi.org/10.4172/2161-0460.1000457

Publisher's Note Springer Nature remains neutral with regard to jurisdictional claims in published maps and institutional affiliations.

The online version of the original article can be found at https://doi.org/ 10.1007/s12035-018-1441-x

Walter J. Lukiw

wlukiw@1suhsc.edu

1 Department of Physiology, University of Toronto, Toronto, ON M5S 1A8, Canada

2 Department of Neuropathology, Toronto General Hospital, Toronto, ON M5G 2C4, Canada

3 Russian Academy of Medical Sciences, Moscow 113152, Russia

4 Walsh Research Institute, Naperville, IL 60540, USA

5 Alchem Biotek Research, Toronto, ON M5S 1A8, Canada

6 Surrey Place Center, University of Toronto, Toronto, ON M5S 1A8, Canada

7 Department of Obstetrics and Gynecology, Toronto, ON M5S 1A8, Canada

8 Department of Biostatistics, School of Public Health, LSU Health Sciences Center, New Orleans, LA 70112, USA
9 Department of Genetics, Louisiana State University Health Sciences Center, New Orleans, LA 70112, USA

10 Louisiana Clinical and Translational Science Center (LA CaTS), LSU Health Sciences Center, New Orleans, LA 70112, USA

11 LSU Neuroscience Center, Louisiana State University Health Sciences Center, New Orleans, LA 70112, USA

12 Department of Anatomy and Cell Biology, Louisiana State University Health Sciences Center, New Orleans, LA 70112, USA

13 Department of Pharmacology, School of Pharmacy, Jiangxi University of TCM, Nanchang, Jiangxi 330004, People's Republic of China

14 Department of Neurology, Louisiana State University Health Sciences Center, New Orleans, LA 70112, USA

15 Department of Ophthalmology, Louisiana State University Health Sciences Center, New Orleans, LA 70112, USA 